\title{
Research on Dynamic System Simulation Model and Algorithm Based on Artificial Neural Network
}

\author{
Xiao Shoubai \\ NanChang Institute of Science \& Technology
}

Keywords: Neural network, Dynamic system, System simulation

\begin{abstract}
With the continuous expansion of applications field on the computer system simulation, the problems of system modeling faced has become more and more complex. At present, there are two main problems: First, modeling object is complex, with a variety of uncertainties and nonlinear characteristics difficult to describe accurately; Second, the requirements of the system modeling more and more higher, urgent to improve the capacity of description of the system model, flexibility, universality and intelligence of the modeling approach. Artificial neural network (ANN) is a new theory and technology for the rapid development of computer intelligent research in recent years. It is applied to computer system simulation modeling because it is used to solve the problem without the need to establish accurate physical model and mathematical model in advance. The multi-layer network can approximate any continuous function, and is equivalent to a differential equation (group) to describe the actual system. It is a kind of system modeling method with strong applicability. At the same time, the method has the ability to learn and acquire the knowledge from the environment (e.g. by learning input/output of the typical sample data input / output to the system, to obtain the hidden rules), so it has good adaptability to the system simulation problem.
\end{abstract}

\section{Introduction}

The theory and method of linear system control have been solved perfectly in the field of automatic control, and some achievements have been made for the complicated nonlinear system. However, due to the complexity and time-varying nature of the actual system, especially for certain processes with uncertainty, it is difficult to establish an accurate mathematical description model. Therefore, the traditional methods universally have the problems of difficult to be modeled, low accuracy and difficult to solve. In recent years, intelligent control theory and methods have been paid attention to and developed, to provide an effective way for solving the problem of complex nonlinear dynamic system control. As the neural network has a high degree of non-linear mapping ability, large-scale parallel distributed processing and good adaptive learning mechanism, there is great potential in dynamic system identification and control. Therefore, the research on application of artificial neural network identification control has good adaptability.

In this paper, the commonly used identification control methods were analyzed. Based on the problem of identification control and optimization solution of dynamic systems, the neural network model and learning algorithm are studied, and the neural network is combined with fuzzy logic and evolutionary algorithms. Different neural network models are constructed to realize system identification control and process optimization in this paper. In the system identification, using the most typical BP neural network for the research of nonlinear systems identification. The fuzzy neural network is used for the design of control model. An improved quantum genetic algorithm is used for the optimization and solution on the control model parameters.

\section{Function and Application Fields of System Simulation}

System simulation is a new discipline which began to emerge in the late 1940s and gradually developed. It can be used to construct a system model that can reflect both system characteristics and system research requirements based on situations that the actual system does not exist or exists but cannot be directly studied on this systems, and carry out the research on the concerned system, to reveal the inherent characteristics, operating rules and the relationship between subsystems of the 
existing and future systems, and predict the future. Simulation techniques can play an important role in all stages of system planning, design, analysis and retrofitting. Now, it has become an indispensable and important method for system analysis, research, design and personnel training.

With the development of simulation technology, the purpose of simulation technology application tends to be diversified and comprehensive. The initial simulation technique was used as the tool for experiments of actual system, and then used to training. Current simulation system application includes the research of system concept, systematic feasibility study, system analysis and design, system analysis and design, system development, system testing and evaluation, system operator training, system prediction, system application and maintenance, and other aspects. Its application fields have been developed to the military and various important areas related to the national economy.

In the military field, simulation is based on the development of weapons and equipment, military training, advanced concepts and military requirements analysis and other aspects; In industrial field, it plays a major role in the process of the concept research and the requirement analysis before the construction of the large-scale construction projects, but also it can optimize the operation of power systems, training of operators in nuclear power plant, and the use of virtual manufacturing technology to solve the problem for manufacturing enterprises on how to develop acceptable product for customers in the shortest possible time and the most economical means. Providing services for combat training of weapon systems and industrial process Simultaneously, the applications of simulation services are being extended to many fields such as transportation, education, communication, society, economy and entertainment. In addition, it also get a great application in the field of oil.

\section{Artificial Neuron Network}

Process Neural Network is one kind of network composed by several neutrons with a certain of topology structure. Similar to the traditional neural network, process neural networks can be divided into feed-forward neural networks and feed-back process neural networks according to the connection method between neurons. Firstly, we define a process neural network model whose weight function can be expanded by a set of basis functions. Assuming that the weight function of the process neural network can be expanded by the basis function $b(t)$, that is, the form of the weight function is limited to a class of simpler function classes, to consider the problem. The basis function $b(t)$ can be a finite basis or a countable basis, and may be orthogonal or non-orthogonal.

$$
\begin{aligned}
& w_{i}(t)=\sum_{k=1}^{i} w_{i k} b_{k}(t) \\
& c_{i}=\int_{0}^{T} w_{i}(t) x_{i}(t) d t \\
& y=f\left(\sum_{i=1}^{n} c_{i}-\theta\right)
\end{aligned}
$$

Where $\theta$ is the threshold value of the fourth layer unit and $\mathrm{f}$ is the excitation function of the fourth layer. Combine $(1 \sim 3)$ together, the relationship between the output and the input process is as follows:

$$
\mathrm{y}=f\left(\sum_{i=1}^{n} \sum_{k=1}^{L} w_{i k} \int_{0}^{T} b_{k}(t) x_{i}(t) d t-\theta\right)
$$

Consider the following combined model of projection, as shown in Fig. 1: 


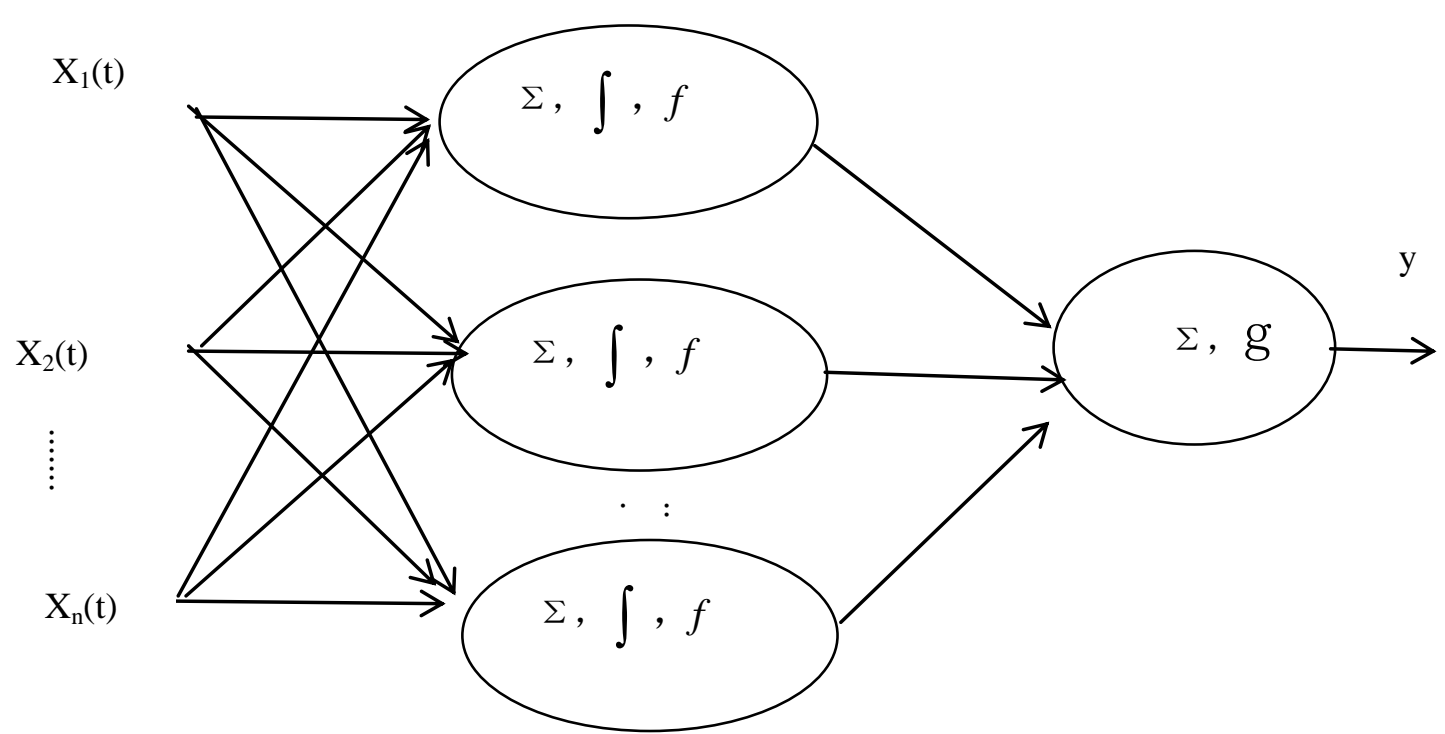

Figure 1. Neural network model of projection combined process

Finally the results are output at the output layer, that is

$$
\mathrm{c}_{i}=\int_{0}^{T}\left(\sum_{j=1}^{n} w_{j i} x_{j}(t)\right) b_{i}(t) d t=\sum_{j=1}^{n} w_{j i} \int_{0}^{T} x_{j}(t) b_{i}(t) d t
$$

\section{Dynamic System Control Based on Feed-forward Process Neural Network}

Artificial neural networks have the nonlinear transformation mechanism and learning ability, as well as high parallel computing power and fault tolerance, providing an effective tool for the identification and control of nonlinear systems. At present, many scientists have applied artificial neural network technology to the control of complex systems. For example, using time-lapse neural network, partial feedback network and other models to identify and control the nonlinear systems. However, when these neural control models solve the problem of system process input / output and time sequence dependency, it mainly realize the delay and correspondence between the input and output of the system by the external delay link, that is to form a time discretization cycle network, is a non-time-varying information processing model essentially. At the same time, the relationship between model structure and the information transfer is complex, which brings some unpredictable problems to the construction and stability of the network learning algorithm, and it is also difficult to reflect the time accumulation effect of the dynamic system process input.

Applying the feed-forward process neural network, a nonlinear dynamic system control model and method are proposed based on process neural network. System process control based on process neural network is used to design process control model based on process neural network on the basis of process identification by applying the adaptive learning mechanism of time-varying environment data and the nonlinear modeling ability under dynamic system study.

Based on the time-varying input / output data of the dynamic system, an identification model based on the process neural network can be established to reflect the forward kinetic characteristics of the system. Then, the system can control the output signal and identification model to establish the reverse control model, to achieve the actual system of process control.

The solution based on system control signal of the process control neural network can be divided into two stages: the first stage is to determine the positive model of the system, namely using system training function sample to confirm identification model of process neural network to meet the positive input / output of actual system; the second stage is to solve process control signal the system to meet the control performance requirements. The solving method of the control model is 
partially based on the robustness and global search ability of the quantum genetic algorithm, to provide the optimization method of the process neural network control model based on the quantum genetic algorithm, and realize the concrete steps of the system process control, and The validity of the model and algorithm is verified by experimental results.

\section{Conclusion}

As the controlled object becomes more and more complex, it shows the characteristics of intricately uncertain process, non-linearity and time-varying, difficult to model accurately, and some models can be established roughly, but faced with the difficulties to solve, which bring more and more highly requirements on control system. Neural network control is a discipline formed by the combination of neural network and automatic control. As artificial neural network has a certain of self-learning, adaptive and nonlinear mapping ability, the application of neural network in the control field has become an inevitable trend of development, and achieved a lot of important results. In this paper, the basic concepts and principles of system identification control are summarized, and the application of feed-forward neural network in nonlinear system identification is introduced. Because of the characteristics of fuzzy neural network itself, it has been widely used in system control. In this paper, fuzzy neural network is used to applied to design the control model, and an improved quantum genetic algorithm is used to optimize the control parameters. In the inverted pendulum control experiment, a good control effect has been achieved.

Project Supported by. Nanchang Key Laboratory of Intelligent Building Network Engineering

\section{References}

[1] Mellit A, Benghanem M, Kalogirou S A. Modeling and simulation of a stand-alone photovoltaic system using an adaptive artificial neural network: Proposition for a new sizing procedure[J]. 2007, 32(2):285-313.

[2] Bergmann S, Stelzer S, Strassburger S. On the use of artificial neural networks in simulation-based manufacturing control[J]. Journal of Simulation, 2014, 8(1):76-90.

[3] Song X M, Kong F Z, Zhan C S, et al. A Hybrid Optimization Rainfall-Runoff Simulation Based on Xinanjiang Model and Artificial Neural Network[J]. Journal of Hydrologic Engineering, 2012, 17(9):1033-1041.

[4] Chen S L. Simulation Model and Algorithm Study of Artificial Neural Network Based Dynamic System[J]. Coal Technology, 2012.

[5] Ming C, Hui Y Z, Ming Z G, et al. Simulation based on artificial neural network for SBBR shortcut nitrification treatment[J]. Zhongguo Huanjing Kexue/china Environmental Science, 2008, 28(8):694-698.

[6] Dong Q U. Simulation of State Equation Identification Modeling for Dynamic Systems Based on Artificial Neural Network (ANN)[J]. Computer Simulation, 2006.

[7] Huang X G, Yu-Chen H U, Huang J R, et al. Study on dynamic simulation system of natural oak growth based on artificial neural network[J]. Journal of Henan Agricultural University, 2013.

[8] Qin R, Yan D, Zhou X, et al. Research on a dynamic simulation method of atrium thermal environment based on neural network[J]. Building \& Environment, 2012, 50(2):214-220.

[9] M. Valčić, J. Skenderović. Identification and simulation models of operating systems based on artificial neural networks[J]. Pomorstvo, 2005, 19(1):43-64. 
[10]Editor $\mathrm{M} \quad \mathrm{K} \quad \mathrm{W}$. E-healthcare systems and wireless communication[C]// Sino-Foreign-Interchange Conference on Intelligent Science and Intelligent Data Engineering. 2012:817-824.

[11]Xu L, Xiao J. Dynamic Modeling and Simulation of PEM Fuel Cells Based on BP Neural Network[C]// International Workshop on Intelligent Systems and Applications. IEEE, 2011:1-3.

[12] Sohn J H, Baek W K. Vehicle dynamic simulation including an artificial neural network bushing model[J]. Journal of Mechanical Science and Technology, 2005, 19(1):255-264. 